\title{
Belgeo
}

Revue belge de géographie

$1 \mid 2019$

Miscellaneous

\section{La Grande Niaye de Pikine, un espace humide à haute valeur agronomique au cœur de l'agglomération urbaine de Dakar : analyse des enjeux socioéconomiques}

The Great Niaye of Pikine, a wetland of high agronomic value in the heart of the urban agglomeration of Dakar: analysis of socio-economic issues

Khalifa Diop, Cheikh Ahmed Tidiane Faye et Seydou Alassane Sow

\section{(2) OpenEdition}

Journals

Édition électronique

URL : http://journals.openedition.org/belgeo/31319

DOI : 10.4000/belgeo.31319

ISSN : 2294-9135

Éditeur :

National Committee of Geography of Belgium, Société Royale Belge de Géographie

\section{Référence électronique}

Khalifa Diop, Cheikh Ahmed Tidiane Faye et Seydou Alassane Sow, «La Grande Niaye de Pikine, un espace humide à haute valeur agronomique au cœur de l'agglomération urbaine de Dakar : analyse des enjeux socioéconomiques », Belgeo [En ligne], 1 | 2019, mis en ligne le 21 janvier 2019, consulté le 10 décembre 2020. URL : http://journals.openedition.org/belgeo/31319; DOI : https://doi.org/ 10.4000/belgeo.31319

Ce document a été généré automatiquement le 10 décembre 2020.

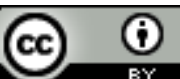

Belgeo est mis à disposition selon les termes de la licence Creative Commons Attribution 4.0 International. 


\section{La Grande Niaye de Pikine, un espace humide à haute valeur agronomique au cœur de l'agglomération urbaine de Dakar : analyse des enjeux socioéconomiques}

The Great Niaye of Pikine, a wetland of high agronomic value in the heart of the urban agglomeration of Dakar: analysis of socio-economic issues

Khalifa Diop, Cheikh Ahmed Tidiane Faye et Seydou Alassane Sow

\section{Introduction}

1 Au Sénégal, la problématique de l'agriculture périurbaine (APU) est abordée en rapport avec son milieu d'évolution qui présente certaines particularités : les Niayes. Celles-ci sont définies comme une zone écologique à haute valeur agronomique formée de dépressions assurant la transition entre les dunes jaunes semi-fixées et le système ogolien continental (dunes rouges). Ces dépressions longent la Grande Côte sénégalaise qui abrite quatre des plus grandes agglomérations du pays: Dakar, Thiès, Louga et Saint-Louis (Fall et al., 2001). Elle "est de loin, la première région économique du Sénégal " (Cissé et al., 2001, p. 10). La production horticole du pays provient à $80 \%$ de cette zone.

2 Cependant, la croissance démographique notée depuis les années 1970 pose de plus en plus la question de l'avenir de cette agriculture. En effet, les Niayes ont connu une croissance démographique très rapide caractérisée par de fortes densités et une extension continue des villes vers les espaces naturels. La population des grandes agglomérations des Niayes $^{1}$ est passée de 2550000 habitants en 1976 à 6709195 
habitants en 2013. La seule région de Dakar compte 3100000 habitants et regroupe plus de $46 \%$ des fonctionnaires sénégalais, $97 \%$ des salariés du commerce et des transports, $96 \%$ des employés de banques, $95 \%$ des entreprises industrielles et commerciales et $87 \%$ des emplois permanents (ANSD, 2013).

3 Selon Diongue (2012) et Diongue et Sakho (2014), cette situation est la conséquence d'une métropolisation impérialiste fondée sur une logique économiste qui tend à drainer l'intérieur du pays au profit des grandes métropoles. Cette première métropolisation qui a imprimé à la capitale sénégalaise une configuration de type centre-périphérie est remplacée par une métropolisation plus polycentrique qui intègre sa périphérie rurale. Ce changement coïncide d'une part avec «le retour de l'État dans la production de l'espace urbain après des décennies marquées par de faibles capacités d'intervention ${ }^{2}$ marqué par les grands projets d'aménagement, notamment dans la périphérie rurale dakaroise; et d'autre part par «la montée en puissance des pouvoirs locaux dans la gestion urbaine " qui est la conséquence de la décentralisation et du transfert de neuf domaines de compétences (dont le domaine, l'aménagement et l'environnement) aux élus locaux (Diongue, 2012).

Dans ce contexte d'explosion démographique et de multiplication des acteurs dans la production de l'espace métropolitain dakarois, les espaces naturels, notamment à vocation agricole, ont subi de fortes mutations.

5 Ainsi, les surfaces horticoles de la région de Dakar auraient baissé de $6 \%$ entre 1988 et 1994. Déjà en 2008, 655 hectares de terres agricoles y ont été convertis en zones d'habitation (Diop, 2012). Dans la Grande Niaye de Pikine en particulier, la dynamique urbaine rapide constitue une menace pour l'avenir de l'agriculture (Diop, 2015).

Cependant, au regard des enjeux que présente le système périurbain de production horticole en matière d'approvisionnement des marchés de la ville, d'emploi des populations urbaines et de recyclage des déchets et effluents urbains, le maintien de la production horticole dans la Grande Niaye de Pikine semble nécessaire. En effet, Dakar est la deuxième région horticole du Sénégal, derrière la région de Thiès. Sa production moyenne annuelle a été évaluée entre 35000 et 40000 tonnes de légumes entre les années 1995 et 2000, soit $25 \%$ de la production nationale (Bâ Diao, 2004). Cette production légumière couvre $60 \%$ de la consommation globale de Dakar, soit 65000 tonnes/an et $35 \mathrm{~kg} /$ habitant/an. L'activité maraîchère dakaroise emploie 15000 personnes dont $60 \%$ de jeunes entre 20 et 39 ans (Guèye et al., 2009). 
Figure 1. Localisation de l'APU dans l'agglomération dakaroise.

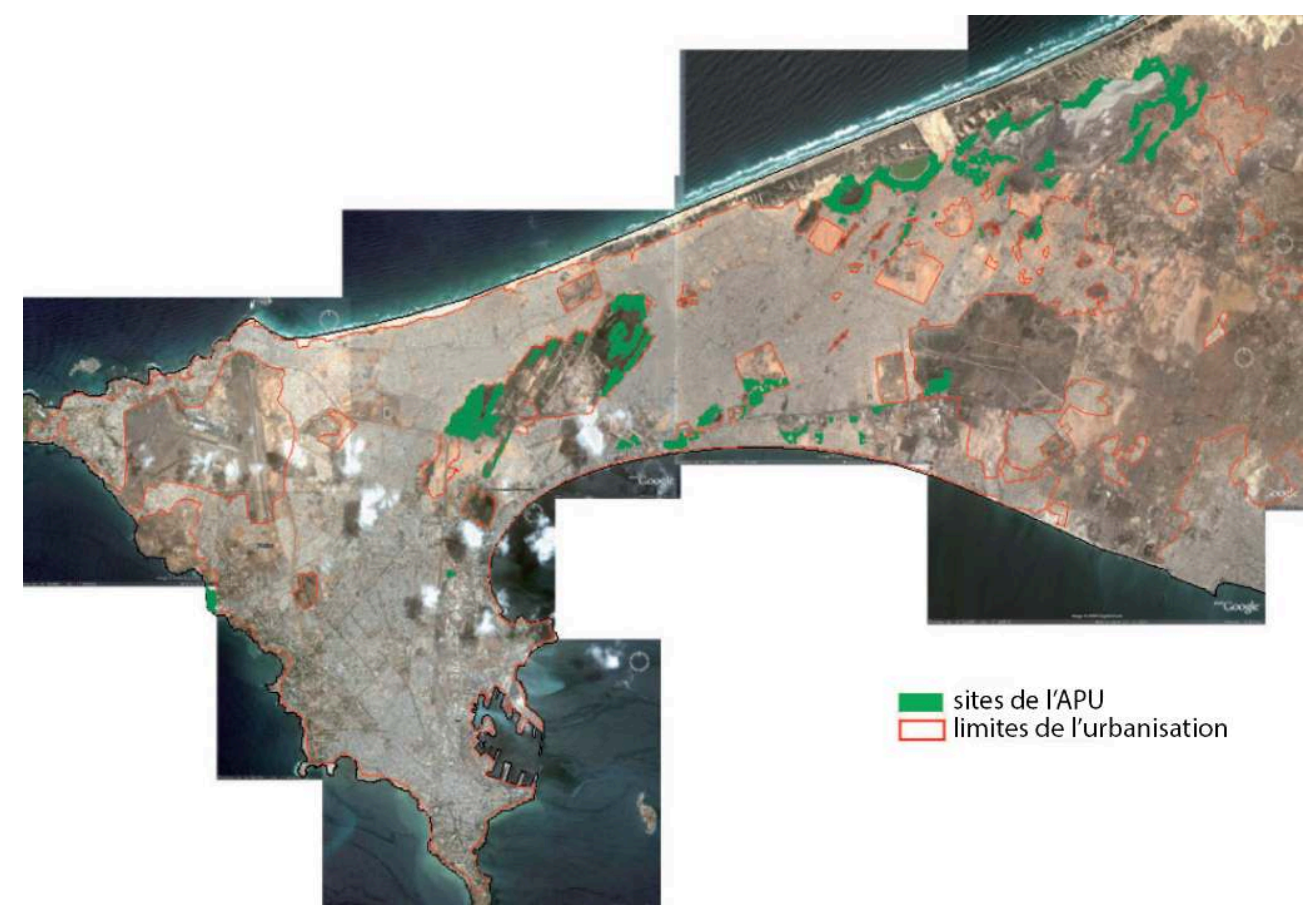

Source : Gaye et Niang, 2010

7 La Grande Niaye de Pikine constitue le premier bassin de production horticole de l'agglomération dakaroise. Elle est essentiellement constituée d'exploitations de moins d'1 hectare. Le maraîchage y est pratiqué toute l'année dans les dépressions et sur les dunes émoussées. Les principales cultures sont la laitue ou salade (Lactuca sativa), qui constitue la culture de prédilection, suivie du piment (Capsicum), du chou (Brassica oleracea), de l'aubergine amère (Solanum aethiopicum), de l'oignon (Allium cepa), de l'aubergine (Solanum melongena L.), de la menthe des champs (Mentha arvensis), etc. L'horticulture y est une opportunité pour «la population urbaine en situation de chômage chronique et les ruraux en migration saisonnière" (Bâ Diao, 2004, p. 4). De plus, cette activité emploie un nombre important de femmes au foyer qui s'activent dans la commercialisation des légumes dans les marchés locaux de Dakar. La proximité de la zone qui garantit la fraîcheur des produits facilite leur écoulement.

8 Le bassin de Pikine a la particularité d'être ceinturé par les agglomérations de Guédiawaye, de Pikine et de Dakar. Celles-ci sont, avec Rufisque, les quatre communes de la région de Dakar. Pikine se situe à l'est de la commune de Dakar, à l'ouest de Rufisque et au sud de la commune de Guédiawaye. C'est son érection en commune par le décret $n^{\circ}$ 90-1134 du 8 octobre 1990 qui sépare Guédiawaye de Pikine (Ndione, 2009). Celle-ci est la ville la plus peuplée de la région de Dakar avec 1000000 d'habitants et 10000 par $\mathrm{km}^{2}$. Elle s'est développée en grande partie dans les Niayes, particulièrement la Grande Niaye de Pikine. 
Figure 2. Localisation de la zone d'étude.

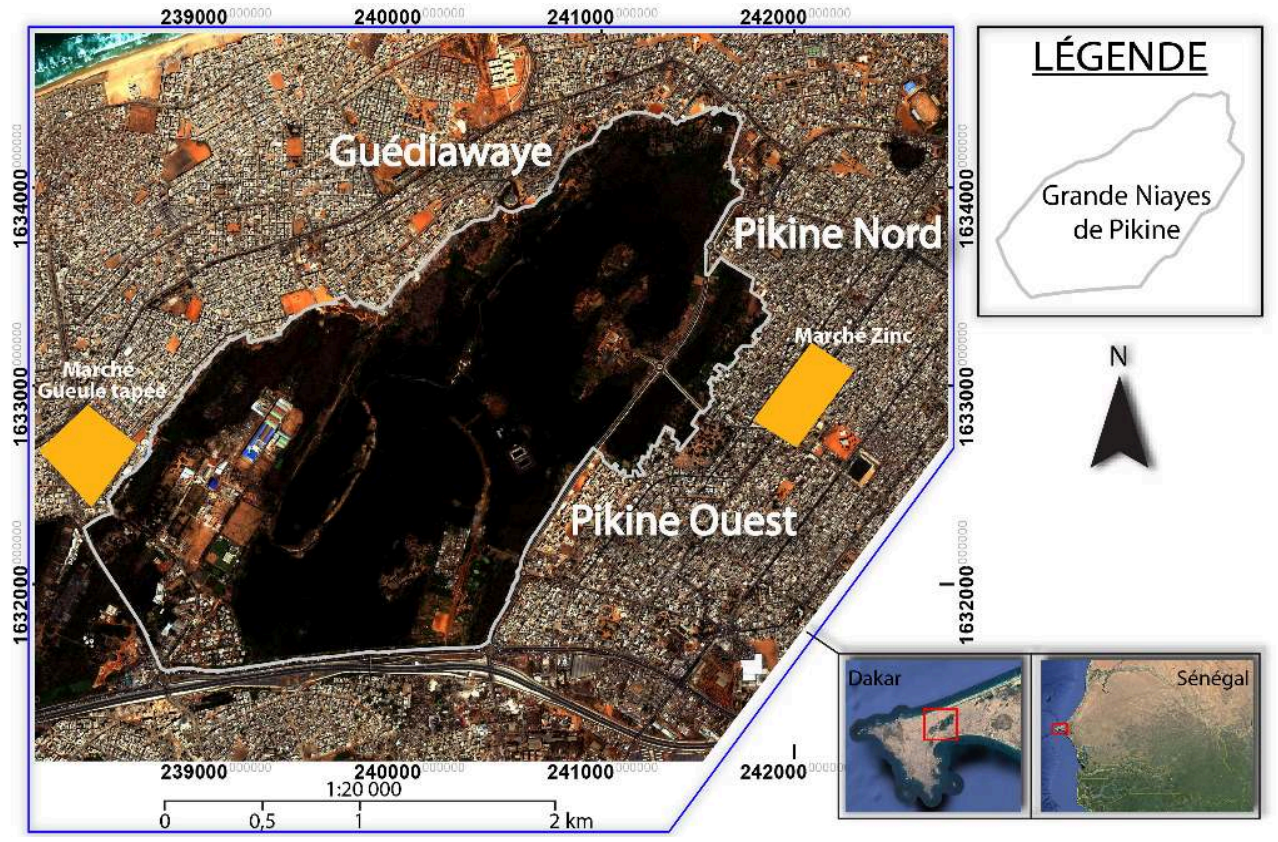

9 Quels sont les enjeux socio-économiques de la production horticole dans la Grande Niaye de Pikine? Telle est la question principale que pose cet article. L'étude cherche d'abord à caractériser la population-cible afin d'établir un diagnostic de la situation socio-économique des acteurs du maraîchage. Ensuite, elle prend en charge l'aspect foncier des parcelles maraîchères dans l'optique de quantifier la régression de celles-ci. Enfin elle tente d'évaluer les risques liés à une éventuelle disparition de la production horticole de la Grande Niaye.

\section{Méthodologie}

Pour quantifier les enjeux de l'APU à Pikine, nous avons procédé à des enquêtes entre le 25 décembre 2017 et le 6 janvier 2018. Elles ont été menées pour répondre à deux objectifs : faire l'état des lieux de la régression des champs maraîchers et évaluer le poids socioéconomique de l'agriculture périurbaine dans la Niaye de Pikine. Pour cela, nous avons ciblé deux populations: les exploitants et les commerçants. Quarante individus ont été interrogés dans le groupe des exploitants sur une population totale de 379 exploitants ${ }^{3}$. Les enquêtés ont été répartis en trois transects correspondant aux différents sites de production maraîchère de la zone : le Technopôle, Pikine nord-Pikine ouest et Guédiawaye. La seconde population regroupe les commerçants de produits maraîchers choisis au hasard dans les deux marchés de proximité de la Grande Niaye : le marché Zinc de Pikine et le marché Gueule Tapée de Cambérène (figure 1). Pour cette population, nous avons procédé par saturation puisqu'il n'existe pas de données officielles de base. Au total, nous avons interrogé 44 commerçants.

11 La population enquêtée compte parmi le groupe d'acteurs qui participe au maintien du système horticole de la Niaye de Pikine. Celui-ci s'est constitué en chaîne impliquant les vendeurs d'intrants et de matériels agricoles, les bineurs, les chaumiers, les exploitants et les vendeuses et vendeurs sur les marchés de proximité. 
12 Pour les exploitants, le questionnaire a d'abord sollicité des renseignements sociodémographiques afin de caractériser cette population. La caractérisation socioéconomique de l'échantillon a permis de connaître l'âge, le sexe, la situation matrimoniale, le niveau de revenu et d'instruction des concernés. Ensuite, les questions ont porté sur le foncier, notamment sur le statut juridique des parcelles, le type de propriété, le mode de tenure en vigueur et les cas d'expropriation. Les résultats obtenus dans ce volet nous ont permis d'apprécier le niveau de précarité foncière des exploitations.

13 Concernant les vendeurs, le questionnaire a ciblé les caractéristiques sociodémographiques des acteurs, mais il a surtout mis en évidence les avantages liés à la proximité du bassin de production et les inconvénients liés à un arrêt éventuel de l'approvisionnement à partir de la Grande Niaye. Ces informations sont déterminantes dans la compréhension des enjeux liés au maintien de l'AUP dans la vallée de Pikine.

\section{Résultats}

14 L'analyse des résultats commence d'abord par la caractérisation et le diagnostic de la situation socioéconomique des acteurs du maraîchage, maraîchers et vendeurs. Ensuite, les conclusions obtenues nous permettront de mesurer le niveau de précarité de la pratique agricole et les risques liés à son éventuelle disparition.

\section{Caractérisation et diagnostic de la situation socio-économique des acteurs du maraîchage}

15 Les résultats des enquêtes ont révélé que la moitié des maraîchers est âgée entre 35 et 49 ans et le tiers entre 18 et 34 ans. Les plus de 50 ans représentent moins $20 \%$, ce qui signifie que la population enquêtée est jeune. Les maraîchers sont en majorité des Wolofs, suivis des Sérères, Peulhs, Diolas et autres (Bambaras, Mandingues). 56 \% de ces individus sont mariés et $48 \%$ ont en moyenne 4 enfants. Plus de la moitié gagne moins de 200000 FCFA $^{4}$ mensuels, soit un peu plus de 6000 FCFA journaliers. $18 \%$ ont des revenus entre 200 et 400000 FCFA par mois et $15 \%$ gagnent entre 600 à 800000 FCFA. La majorité $(70 \%)$ ne dispose pas de sources de revenus parallèles tandis que $29 \%$ affirment en avoir au moins une. Si l'on compare les revenus tirés de l'agriculture à ceux des autres activités, 89 \% disent gagner plus avec le maraîchage contre $11 \%$ qui soutiennent le contraire (figure 3). 
Figure 3. Caractéristiques socioéconomiques des maraîchers de la Grande Niaye de Pikine.

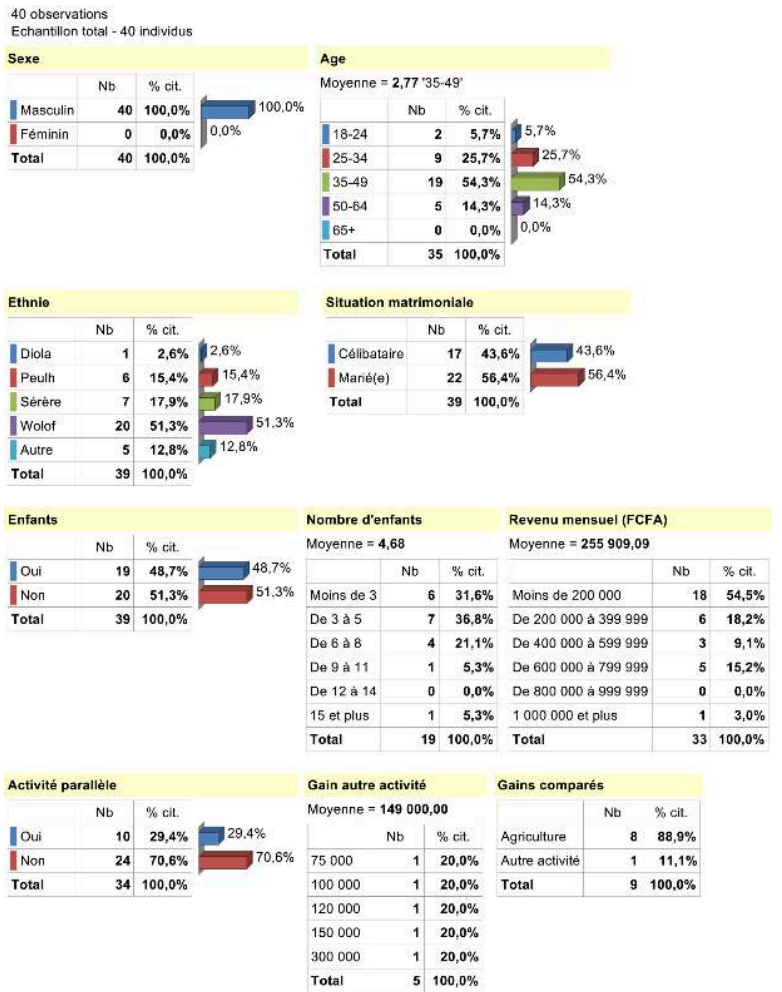

16 Concernant le niveau d'instruction, les maraîchers sont à plus de 75 \% instruits, dont $50 \%$ par l'école française, $20 \%$ par l'école coranique et $10 \%$ par l'école arabe. Les moins de $25 \%$ restants déclarent n'avoir pas reçu d'instruction. Seulement $15 \%$ ont atteint le niveau secondaire contre $35 \%$ du niveau moyen et la moitié est du niveau primaire. Il est à noter que $64 \%$ des instruits ont au moins un diplôme scolaire et $14 \%$ ont le bac mais aucun n'a fait d'études supérieures (figure 4). 
Figure 4. Niveau d'instruction des maraîchers.

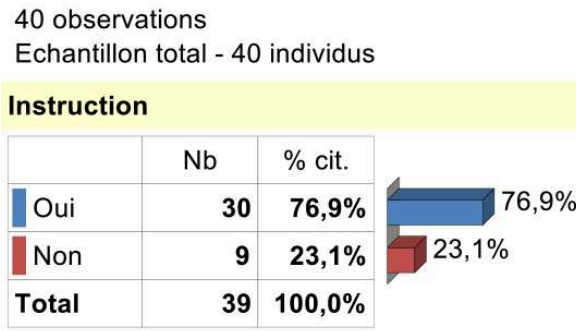

Echantillon total - 40 individus

\begin{tabular}{|c|c|c|c|}
\hline & $\mathrm{Nb}$ & $\%$ cit. & \\
\hline Oui & 30 & $76,9 \%$ & $76,9 \%$ \\
\hline Non & 9 & $23,1 \%$ & $23,1 \%$ \\
\hline Total & 39 & $100,0 \%$ & \\
\hline
\end{tabular}

\begin{tabular}{|c|c|c|c|}
\hline \multicolumn{4}{|c|}{ Niveau d'instruction } \\
\hline & $\mathrm{Nb}$ & $\%$ cit. & \\
\hline Primaire & 10 & $50,0 \%$ & $50,0 \%$ \\
\hline Moyen & 7 & $35,0 \%$ & $35,0 \%$ \\
\hline Secondaire & 3 & $15,0 \%$ & $15,0 \%$ \\
\hline Supérieur & $\mathbf{0}$ & $0,0 \%$ & $0,0 \%$ \\
\hline Total & 20 & $100,0 \%$ & \\
\hline
\end{tabular}

Type d'instruction
\begin{tabular}{|l|r|r|}
\hline & $\mathrm{Nb}$ & $\%$ obs. \\
\hline Ecole coranique & $\mathbf{8}$ & $\mathbf{2 0 , 0} \%$ \\
\hline Arabe & $\mathbf{4}$ & $\mathbf{1 0 , 0} \%$ \\
\hline Ecole française & $\mathbf{2 0}$ & $\mathbf{5 0 , 0} \%$ \\
\hline Total & $\mathbf{4 0}$ & \\
\hline
\end{tabular}

\begin{tabular}{|c|c|c|c|}
\hline \multicolumn{4}{|c|}{ Dernier diplôme } \\
\hline & $\mathrm{Nb}$ & $\%$ cit. & \multirow{2}{*}{$64,3 \%$} \\
\hline CFEE & 9 & $64,3 \%$ & \\
\hline BFEM & 1 & $7,1 \%$ & $7,1 \%$ \\
\hline Bac & 2 & $14,3 \%$ & $14,3 \%$ \\
\hline Licence & 0 & $0,0 \%$ & $0,0 \%$ \\
\hline Master & 0 & $0,0 \%$ & $0,0 \%$ \\
\hline Doctorat & 0 & $0,0 \%$ & $0,0 \%$ \\
\hline Autre & 2 & $14,3 \%$ & $14,3 \%$ \\
\hline Total & 14 & $100,0 \%$ & \\
\hline
\end{tabular}

De manière générale, les maraîchers de la Grande Niaye de Pikine affirment gagner suffisamment pour subvenir aux besoins de leurs familles. Si certains disposent d'un travail leur permettant de hausser leur revenu, la plupart n'exercent que dans l'agriculture. Celle-ci paye mieux que les autres activités selon les trois-quarts de ceux qui ont déclaré en avoir une. Ce sont des personnes généralement instruites dont certaines disposent d'un diplôme scolaire.

Concernant les vendeurs de produits agricoles, près de $98 \%$ de la population interrogée sont des femmes (figure 5). Elles sont relativement jeunes, âgées à plus de $50 \%$ entre 25 et 34 ans. $45 \%$ ont entre 35 et 49 ans et $2 \%$ de ces femmes sont très jeunes (18 - 24 ans). Moins de $5 \%$ sont célibataires alors que plus de $95 \%$ déclarent être mariées. Parmi elles, $95 \%$ ont des enfants et $60 \%$ ont au moins 4 enfants. Elles gagnent en moyenne 130000 FCFA par mois ou 4000 FCFA par jour. Seulement $2 \%$ affirment gagner plus de 250000 FCFA par mois contre $52 \%$ qui gagnent entre 100000 et 150000 FCFA et $36 \%$ qui perçoivent entre 150000 et 200000 FCFA. Ce qui fait que le niveau de revenu est très moyen. Il l'est encore plus si l'on considère que $9 \%$ ne gagnent pas 100000 FCFA par mois, soit 3300 FCFA par jour. 
Figure 5. Caractéristiques socio-économiques des vendeurs de produits de la Niaye de Pikine.

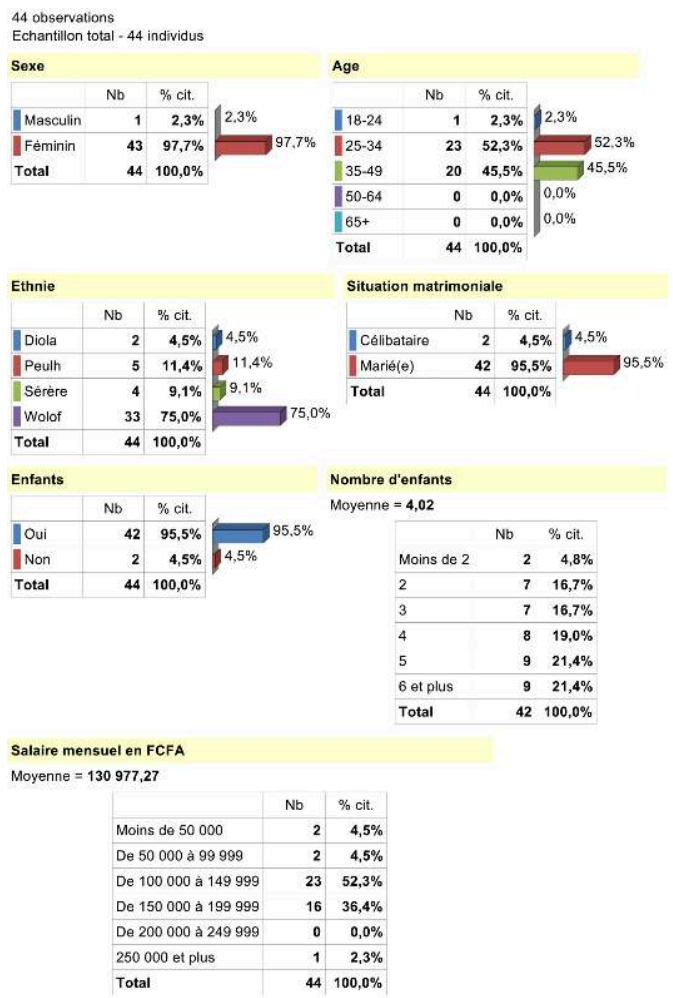

Tous les commerçants interrogés au marché Zinc et de Gueule Tapée s'approvisionnent dans la Niaye (figure 6). Le produit le plus prisé est la laitue (salade) commercialisée par tous les vendeurs. Il est suivi de la tomate achetée par $93 \%$ et du piment acheté par $31 \%$ des interrogés. Ensuite vient l'aubergine $(20 \%)$, le concombre et le jaxatu (aubergine amère). La préférence pour ces produits est due à deux facteurs principaux et interdépendants : la proximité du bassin de production qui assure la fraîcheur des produits et l'accessibilité de leur prix. En effet, les marchés sont situés à moins de $1 \mathrm{~km}$ de la Niaye, accessible à moins de 10 minutes de marche. Ceci fait que près de $98 \%$ des vendeurs ont cité la proximité comme l'atout principal de leur commerce.

Par conséquent, 88 \% confirment que la fraîcheur des produits est un avantage déterminant dans leur activité. Au demeurant, il semble que la proximité n'a qu'un impact limité sur le prix des produits. Seulement $7 \%$ des vendeurs reconnaissent acheter moins cher de la Grande Niaye. Ces enjeux combinés nous ont poussés à nous interroger sur les conséquences d'une éventuelle cessation de l'approvisionnement dû à un facteur quelconque, notamment la conversion des champs en bâti qui empêcherait la pratique convenable du maraîchage.

21 Ainsi, $45 \%$ des vendeurs prévoient des préjudices liés à la distance qu'ils auraient à parcourir pour s'approvisionner dans d'autres bassins de production et $29 \%$ craignent pour la qualité des produits liée à une éventuelle baisse de la fraîcheur. Par conséquent, $23 \%$ envisagent une baisse de la clientèle, qui préfère les produits frais. Cependant, un impact moindre est prévu sur les prix ( $1 \%$ des enquêtés). 
Figure 6. Analyse des avantages du maraîchage selon les commerçants.

44 observations

Echantillon total - 44 individus

Achat de produits de la Niayes

\begin{tabular}{|l|r|r|l|}
\hline & Nb & \multicolumn{1}{|c|}{$\%$ cit. } & \\
\hline Oui & $\mathbf{4 4}$ & $\mathbf{1 0 0 , 0} \%$ & \\
\hline Non & $\mathbf{0}$ & $\mathbf{0 , 0} \%$ & $0,0 \%$ \\
\hline Total & $\mathbf{4 4}$ & $\mathbf{1 0 0 , 0} \%$ & \\
\hline
\end{tabular}

Avantages ?

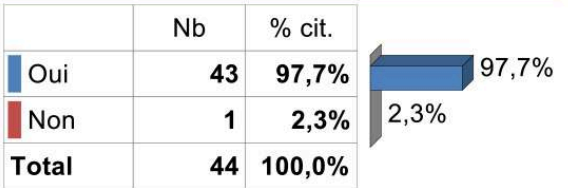

\begin{tabular}{|c|c|c|c|}
\hline \multicolumn{4}{|c|}{ Variétés achetées } \\
\hline & $\mathrm{Nb}$ & $\%$ obs. & \\
\hline Salade & 44 & $100,0 \%$ & $100,0 \%$ \\
\hline Aubergine & 9 & $20,5 \%$ & $20,5 \%$ \\
\hline Jaxatu & 2 & $4,5 \%$ & $4,5 \%$ \\
\hline Concombre & 4 & $9,1 \%$ & $9,1 \%$ \\
\hline Tomates & 41 & $93,2 \%$ & $93,2 \%$ \\
\hline Piment & 14 & $31,8 \%$ & $31,8 \%$ \\
\hline Total & 44 & & \\
\hline
\end{tabular}

Quels avantages?

\begin{tabular}{|c|c|c|c|}
\hline & $\mathrm{Nb}$ & $\%$ obs. & \\
\hline Moins cher & 3 & $7,0 \%$ & \\
\hline Proximité & 42 & $97,7 \%$ & $97,7 \%$ \\
\hline Fraîcheur du produit & 38 & $88,4 \%$ & $88,4 \%$ \\
\hline Total & 43 & & \\
\hline
\end{tabular}

D'une manière générale, l'agriculture périurbaine dans la Niaye de Pikine implique des pères et mères de famille mais également des jeunes femmes et hommes à revenus moyens. La plupart d'entre eux vivent de cette activité. Le paramètre de la proximité semble être le principal atout de ce partenariat producteurs-vendeurs. Ainsi, une perturbation de l'équilibre du système de production pourrait avoir des conséquences non désirables sur toute la chaîne de valeur. Ceci pourrait occasionner un travail plus difficile pour les commerçants comme le craint la majorité de ceux enquêtés. Cela semble être le principal enjeu de la gestion de la Niaye qui continue de régresser sous la pression urbaine.

\section{Statut foncier et coût socio-économique des parcelles maraîchères menacées}

Les questions ont porté sur la propriété, la tenure, le mode de faire-valoir et le statut juridique des parcelles. Nous remarquons que $59 \%$ ne sont pas propriétaires des parcelles qu'ils cultivent. Parmi les propriétaires, $87 \%$ soutiennent détenir une pièce justificative de leur propriété. Près de $75 \%$ disent disposer d'un permis d'occuper pour $25 \%$ qui disent être en possession d'un bail. Concernant la tenure, $45 \%$ des enquêtés propriétaires déclarent exploiter leur propre terre sans précisions supplémentaires et $20 \%$ révèlent qu'ils en disposent par héritage. Les modes de faire-valoir sont dominés par l'exploitation personnelle ou familiale (66\%). Le métayage est pratiqué à hauteur de $20 \%$ suivi de la location à $11 \%$ et du prêt à près de $3 \%$.

Globalement, seuls $6 \%$ des parcelles maraîchères semblent en règle selon les données collectées. Si la majorité des producteurs trouvés sur place affirment ne pas être propriétaires, ils déclarent cependant qu'ils sont soit sur la propriété d'une famille, soit bénéficiaires d'un mode de faire-valoir. Nous précisons que les informations sur le statut juridique des parcelles ont été obtenues pour une large part par l'intermédiaire de non propriétaires. Ceci nuance quelque peu ces résultats mais les valide en quelque sorte. En effet, la non-propriété est interprétée comme le fait de ne pas détenir les 
papiers légaux de la parcelle. Toutefois, nous avons précisé que nombre des enquêtés travaillent sur la propriété de parents dont ils affirment être proches. C'est sans doute pourquoi ils peuvent avoir des informations sur la régularité ou non du terrain.

Figure 7. Statut foncier des exploitations agricoles.

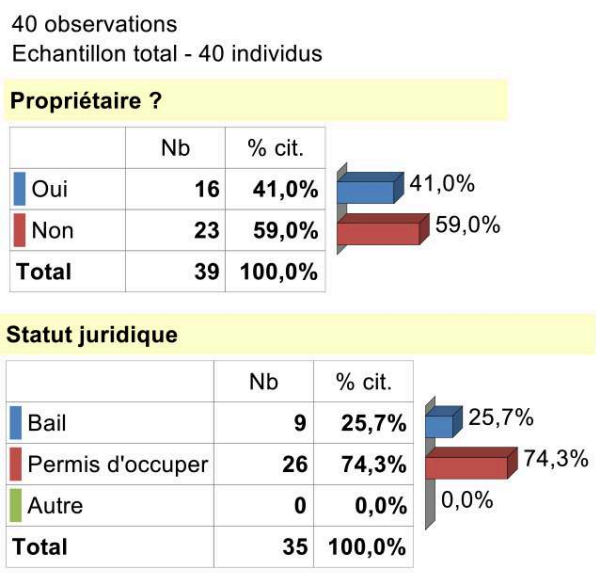

\begin{tabular}{|c|c|c|c|}
\hline & $\mathrm{Nb}$ & $\%$ cit. & \\
\hline Propriété & 16 & $45,7 \%$ & $45,7 \%$ \\
\hline Héritage & 7 & $20,0 \%$ & $20,0 \%$ \\
\hline Location & 4 & $11,4 \%$ & $11,4 \%$ \\
\hline Prêt & 1 & $2,9 \%$ & $2,9 \%$ \\
\hline Métayage (bay seddoo) & 7 & $20,0 \%$ & $20,0 \%$ \\
\hline Autre & 0 & $0,0 \%$ & $0,0 \%$ \\
\hline Total & 35 & $100,0 \%$ & \\
\hline
\end{tabular}

Concernant les expropriations, $37 \%$ des enquêtés affirment en avoir été victimes, tandis que plus de $60 \%$ disent ne pas avoir subi le phénomène. L'enquête a révélé que les expropriations ont été plus fréquentes entre 2011 et 2016. Parmi les causes les plus citées on retrouve la construction d'une autoroute et de l'arène nationale. Ces deux chantiers ont touché respectivement $70 \%$ et $30 \%$ des expropriés durant cette période. La plupart des maraîchers déchus sont dédommagés (78\%), mais $21 \%$ disent ne pas l'avoir été. Les dédommagements s'élèvent en moyenne à 687000 FCFA, dissimulant toutefois des disparités. En effet, $30 \%$ affirment avoir reçu moins de 300000 FCFA, $30 \%$ ont obtenu entre 300000 et 599000 FCFA, $10 \%$ entre 600000 et 900000 FCFA et $30 \%$ ont touché 1500000 FCFA ou plus. Les dédommagements ne satisfont pas tous les maraîchers. 40 \% de ceux qui ont été expropriés ont déclaré avoir refusé de céder leurs terres. $7 \%$ ont affirmé avoir abandonné l'activité pour y revenir quelque temps après. 
Figure 8. Analyse de l'expropriation foncière.

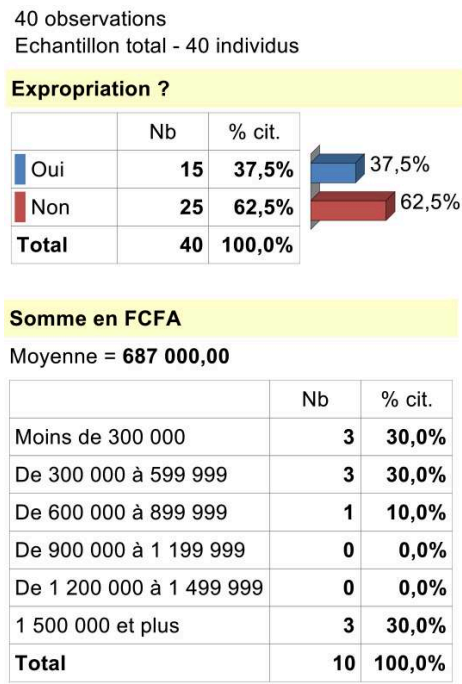

Réaction

\begin{tabular}{|l|r|r|}
\hline & Nb & $\%$ obs. \\
\hline Résistance & $\mathbf{6}$ & $40,0 \%$ \\
\hline Chercher une autre parcelle & $\mathbf{0}$ & $0,0 \%$ \\
\hline Abandon de l'activité & 7 & $46,7 \%$ \\
\hline Autre & 2 & $13,3 \%$ \\
\hline Total & 15 & $100,0 \%$ \\
\hline
\end{tabular}

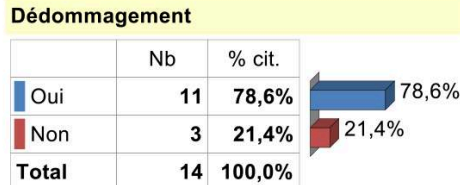

\begin{tabular}{ll|l|l} 
Total & 14 & $100,0 \%$
\end{tabular}

L'analyse de l'expropriation foncière révèle que les pertes de terres agricoles au profit d'aménagements non agricoles restent très actuelles (figure 9). Les expropriations peuvent être brutales et souvent difficiles à prévoir par les producteurs.

Figure 9. Remblai d'une ancienne exploitation au Technopôle.

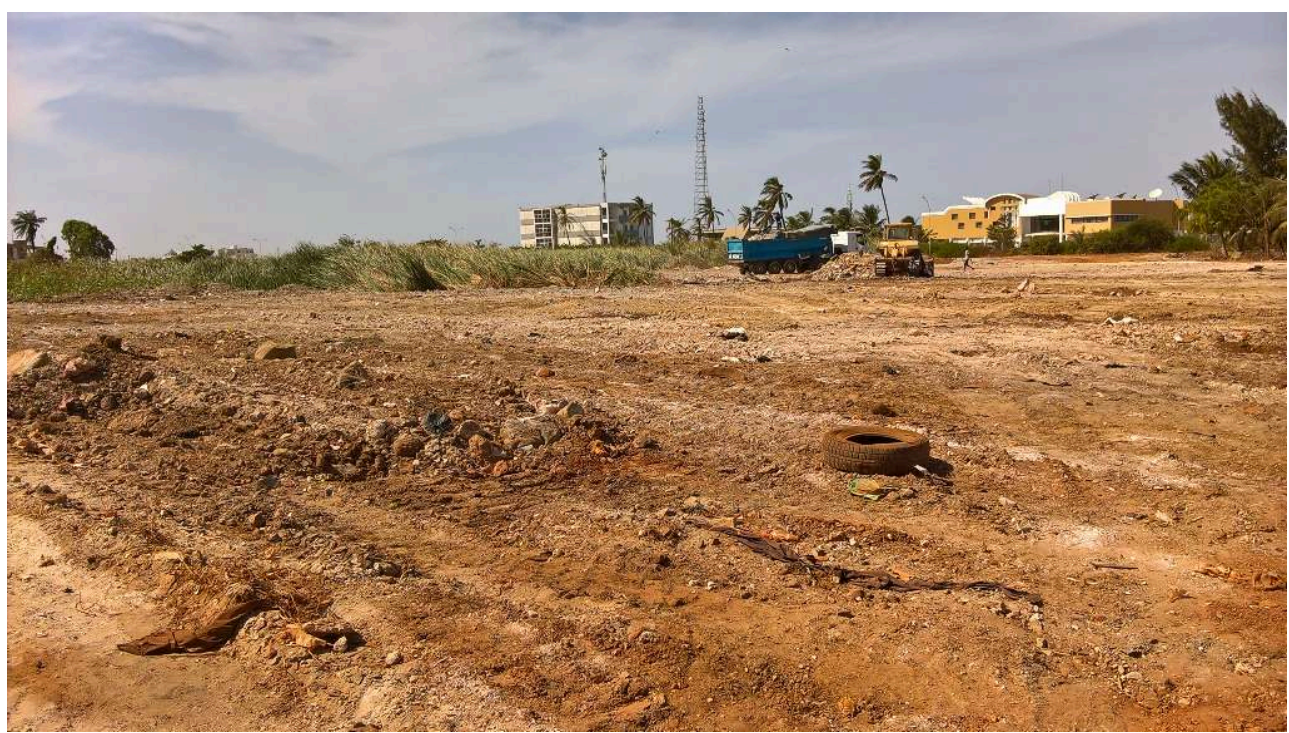

Cliché : Diop (déc. 2016)

La figure 9 témoigne de l'actualité de l'expropriation foncière à Pikine, notamment au Technopôle. Selon les enquêtés, les terrains remblayés sont destinés à accueillir des établissements commerciaux. La pression est d'autant plus importante qu'elle ne se 
limite pas uniquement au Technopôle, mais concerne toute la Niaye. A Pikine Nord, l'installation des établissements commerciaux, d'ateliers (figures 10 et 11) et d'édifices à but ludique est fréquente. Les conversions d'espaces concernent aussi bien les bords que l'intérieur de la Niaye.

Figure 10. Garage avoisinant un champ à Pikine Ouest.

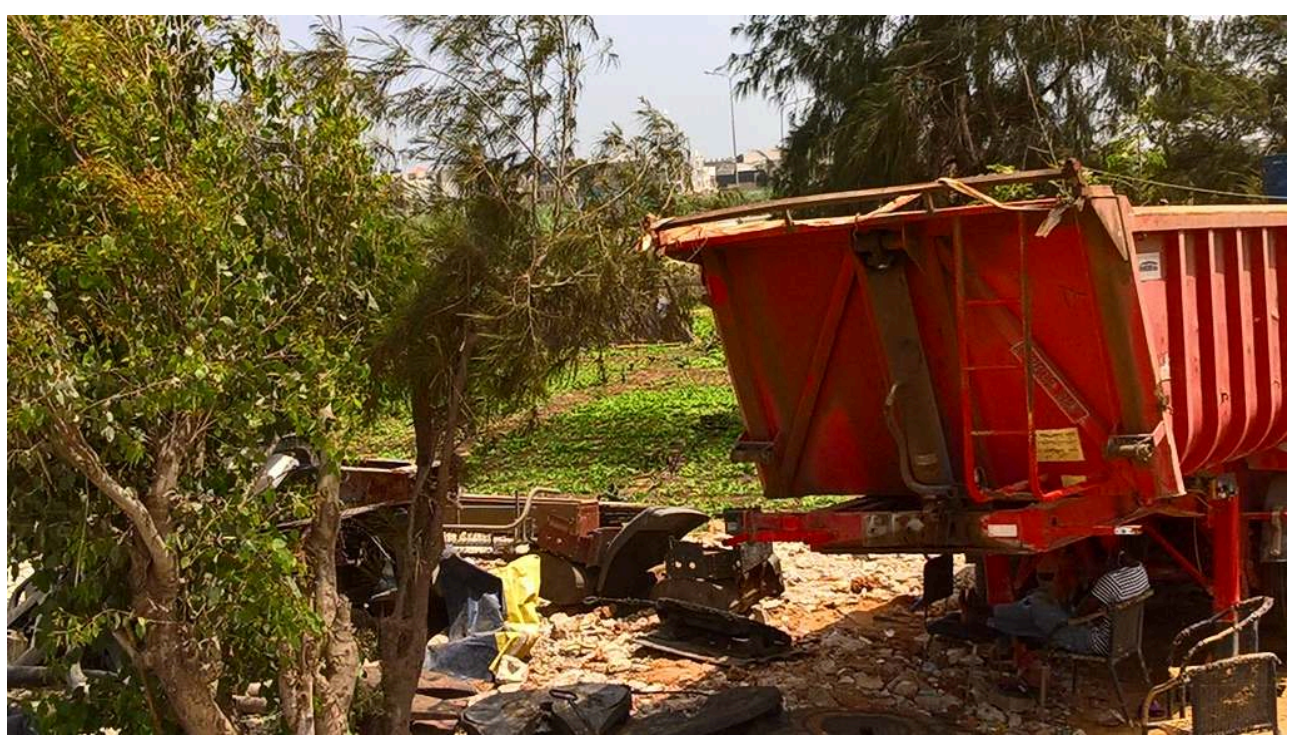

Cliché : Diop (27 mai 2017)

Figure 11. Garage installé sur un ancien champ à Pikine Ouest.

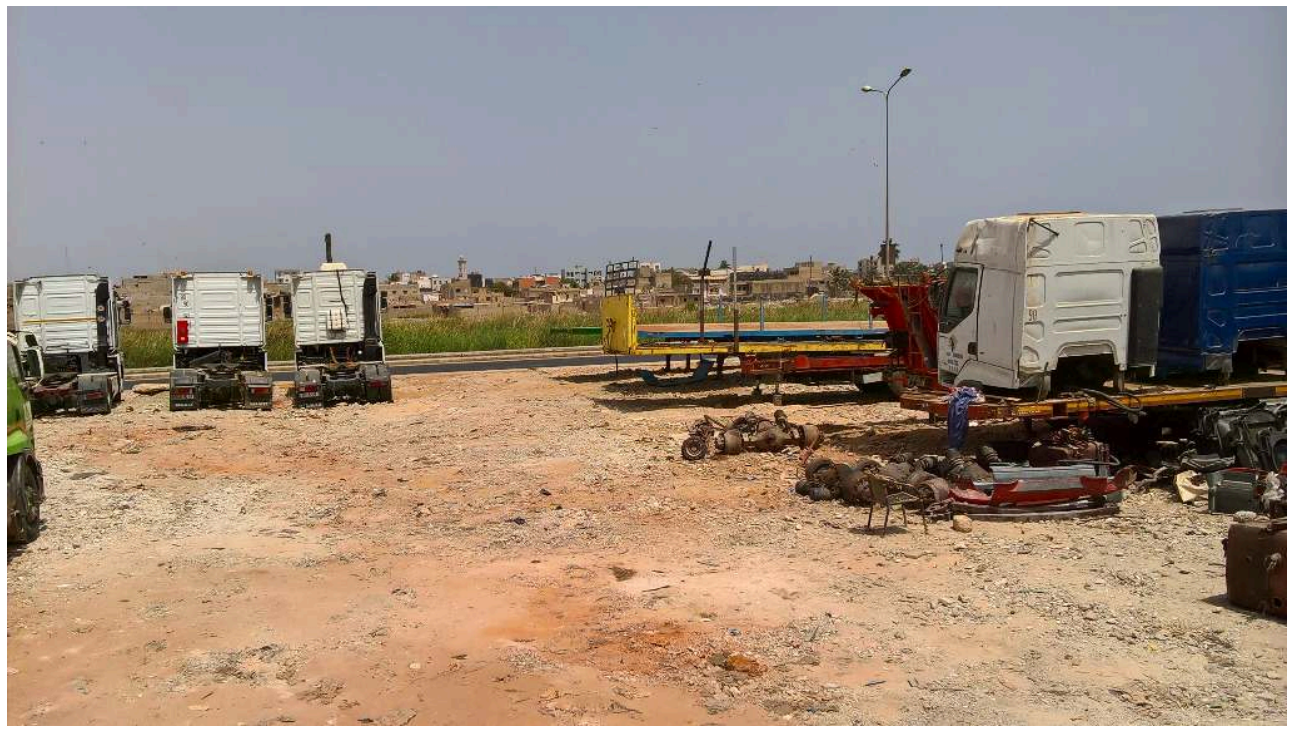

Cliché : Diop (27 mai 2017)

Les images montrent des conversions de terres agricoles le long de l'axe 4 du PRECOL ${ }^{5}$. Celui-ci étant un aménagement structurant, il en attire d'autres voulant bénéficier de plus de visibilité. Cette conjoncture accentue les spéculations sur les exploitations bordant la route. Ce même phénomène est observé avec la construction de l'arène nationale (figure 12) qui commence à attirer d'autres aménagements alors qu'elle n'est même pas encore fonctionnelle. 
Figure 12. Transformation spatiale autour de l'arène nationale.

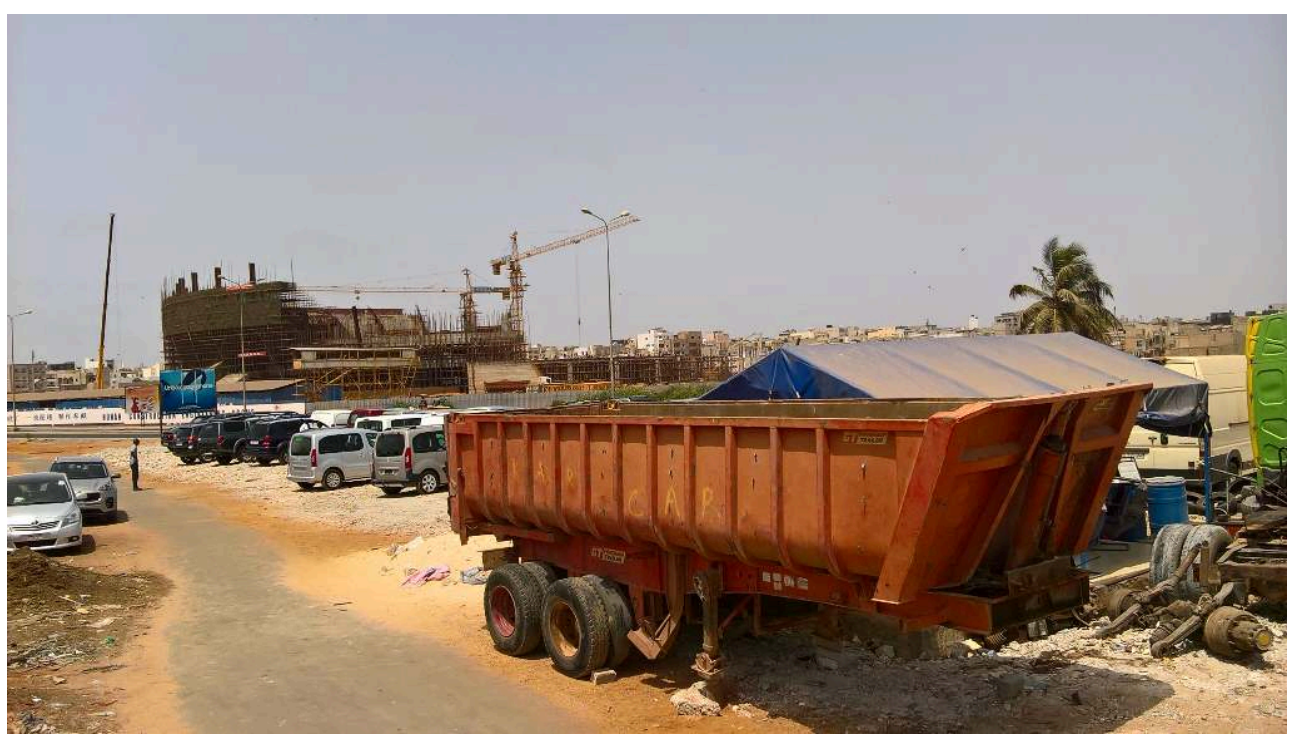

Cliché : Diop (27 mai 2017)

\section{Discussion}

La baisse des superficies cultivables au profit de l'urbain à Pikine s'inscrit dans la dynamique d'étalement de l'agglomération de Dakar. Les travaux de Diop (2006) confirment cette étude à Pikine mais aussi à Yeumbeul, anciennes cuvettes devenues la deuxième banlieue de Dakar. L'essentiel de ces habitations a pris pied dans des zones non aedificandi, ce qui accentue les impacts des inondations qui ont gagné en ampleur depuis les retours pluvieux en 2006.

Ce contexte pose en même temps la question de l'assainissement à cause de la proximité de la nappe et l'absence de réseaux de drainage des eaux pluviales. Cette situation a tendance à toucher la majeure partie de la banlieue dakaroise. Ndao (2012) a fait l'état des lieux à Djeddah Thiaroye Kao qui intègre la même problématique. Il faut noter aussi que la sollicitation de ces espaces ne se limite pas à Dakar, elle concerne toute la frange côtière bordée par les Niayes. L'attractivité du milieu (biodiversité et climat subguinéen) est considérée comme un facteur important de l'urbanisation de la zone.

31 Sur le plan de l'aménagement du territoire, la réduction des parcelles maraîchères de la Grande Niaye de Pikine suscite la question de l'efficacité de la gestion de cet espace. Bien que protégée par le Programme d'Actions pour la Sauvegarde et le Développement Urbain des Niayes et zones vertes de Dakar (PASDUNE) ordonné par le décret $n^{\circ}$ 2002-1042 du 15 octobre 2002 du Ministère de l'Urbanisme et de l'Habitat de l'époque, la zone continue de recevoir la ville (figure 13). 
Figure 13. Extension irrégulière de la ville autour de la Niaye de Pikine.

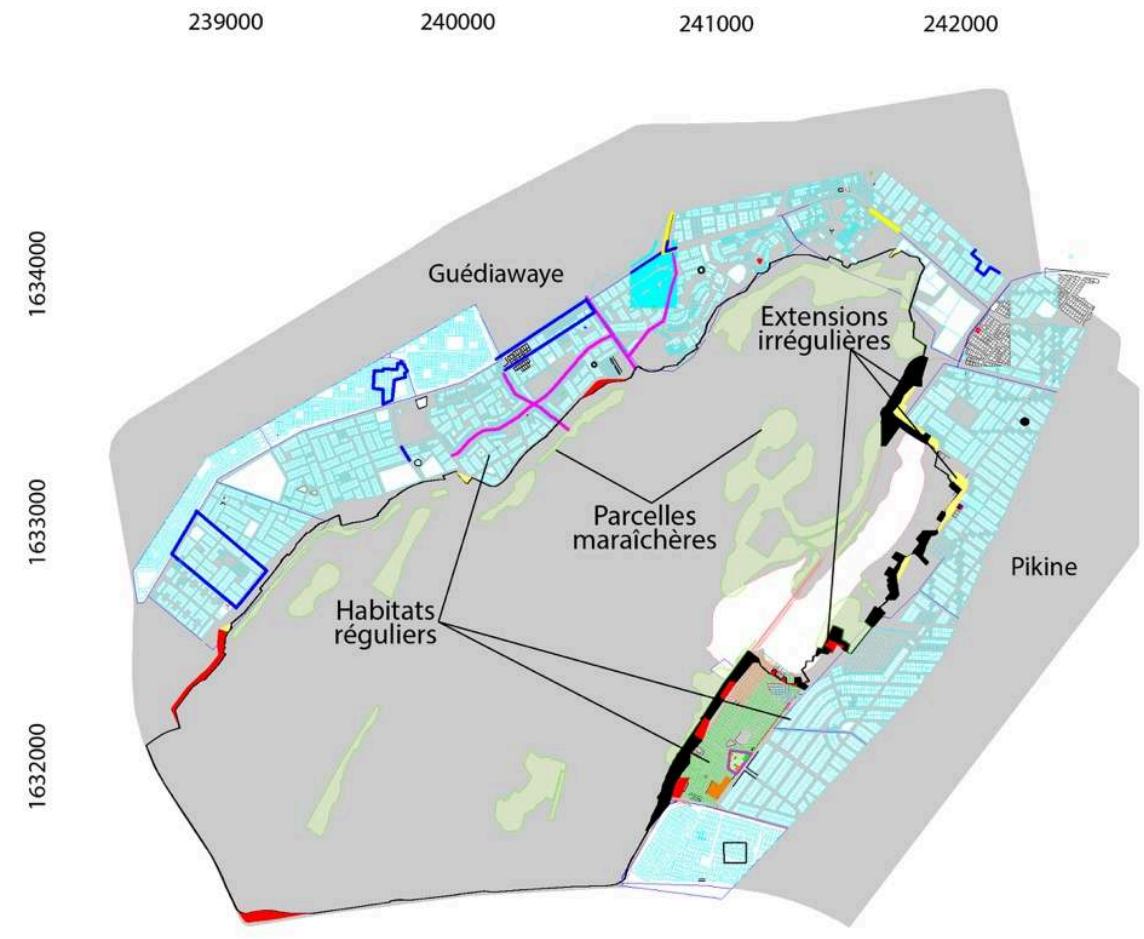

Source des données : Service cadastral de Pikine (2015)

Ainsi, les résultats obtenus par l'étude ne seraient pas le fait de la seule continuité d'une urbanisation soutenue par la croissance démographique, mais elle découlerait aussi d'une application peu rigoureuse des mesures de conservation prises. Ndao (2012) précise à cet effet que ce programme n'a pas abouti faute de "mécanisme de concrétisation » (p. 219). Elle attribue ce blocage aux conflits d'intérêts entre acteurs et aux contraintes juridiques et institutionnelles. En effet, explique-t-elle, chaque catégorie d'acteurs (maraîchers, entrepreneurs, promoteurs immobiliers) perçoit l'espace selon ses besoins. Ainsi, il est difficile de préserver les Niayes si l'on veut leur attribuer une seule fonction. De plus, il existe des lenteurs administratives qu'elle attribue à un manque de volonté de la part des hommes politiques.

Également, le statut foncier de cet espace participe pour beaucoup à sa fragilité. Le statu quo est en effet une superposition de possessions datant de l'avant-colonisation jusqu'à la dernière loi sur le domaine national de 1964. Le régime coutumier basé sur la collectivité des terres indivisibles et inaliénables géré par le lamane ${ }^{6}$ a été perturbé par l'avènement du Code civil français de septembre 1830. Ce dernier consacre la propriété individuelle et instaure le régime de l'immatriculation le 26 juillet 1932. La tenure est désormais sur fond de reconnaissance administrative à travers l'immatriculation : pas de droit réel sans immatriculation. Cette mesure s'est heurtée à la résistance des chefs coutumiers au point qu'en 1960, le nouveau régime indépendant s'est vu dans l'obligation de réinventer la législation foncière du pays.

C'est dans ce cadre qu'a été instauré un régime dualiste alliant droit de propriété et de non-propriété. Mais en 1964, la loi 46-64 sur le domaine national est venue annihiler le droit coutumier de possession et a transféré toutes les terres non immatriculées ou pas en procédure d'immatriculation au seul compte de l'État. Cette fois aussi, cette loi a 
rencontré la résistance des lamanes qui se réclament toujours maîtres de leurs terres. Certains ont profité du Code civil français pour faire constater par actes administratifs leurs possessions, bien que la loi 46-64 stipule que ce type de possession n'est rattaché à aucun patrimoine ou propriété.

La Grande Niaye de Pikine a hérité de cette hétérogénéité de tenures. En effet, on y trouve des possessions de l'État, conformément à la loi de 46-64, mais aussi de privés. Le domaine du Technopôle est une propriété de l'État, de même que la grande majorité des terres du domaine du $\mathrm{CDH}$, la partie restante étant détenue par des privés, qui possèdent aussi la partie sud du Technopôle. Le Golf club de Dakar, situé dans la partie Nord du Technopôle et la Cité Fayçal de Cambérène sont également détenus par des privés (figure 14).

Figure 14. Répartition du foncier de la Grande Niaye.

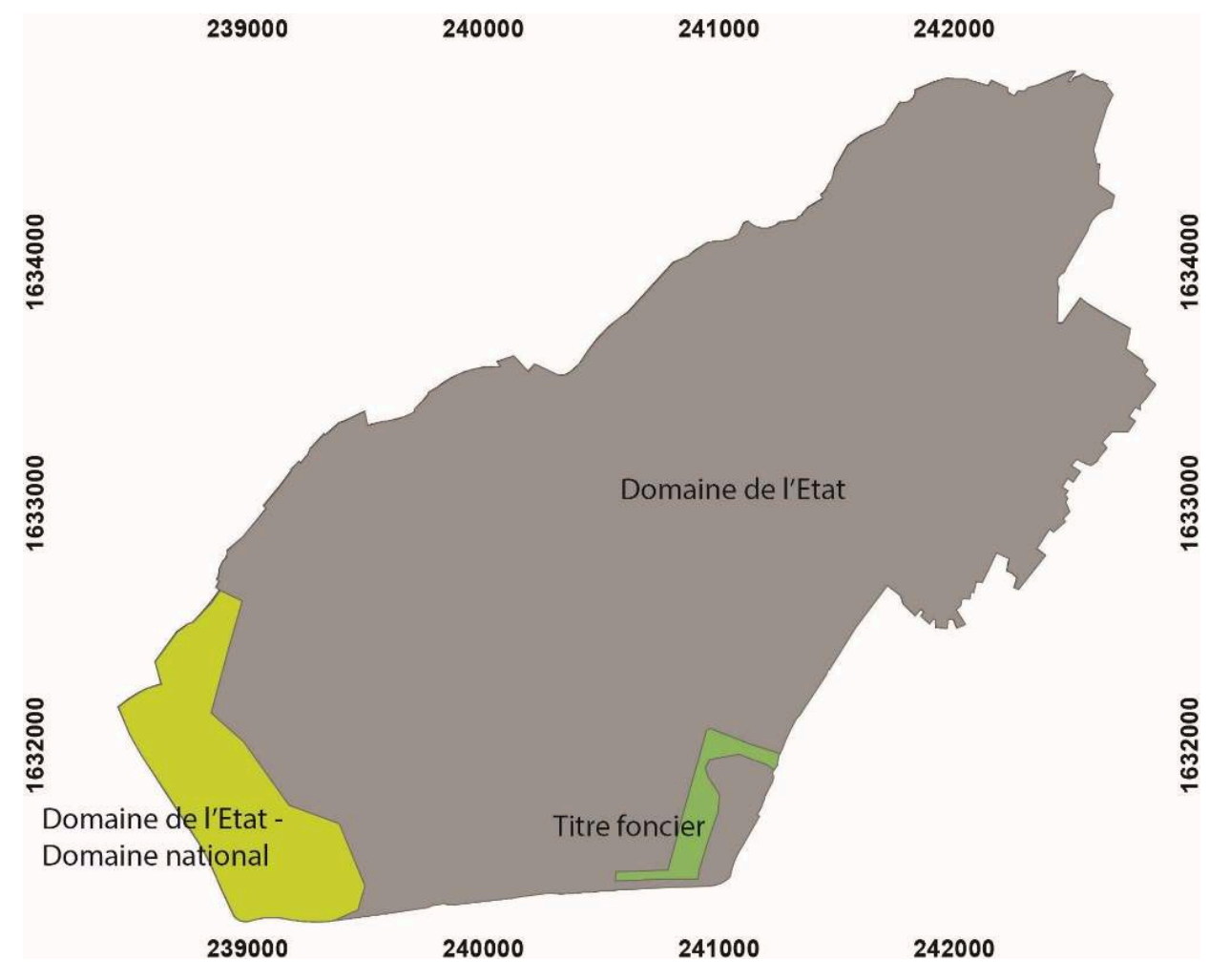

Source : PASDUNE 2002

Au-delà de la Grande Niayes de Pikine, l'étude des enjeux de l'agriculture périurbaine coïncide avec l'intérêt que suscitent les débats sur la durabilité des systèmes alimentaires face à l'urbanisation. Celle-ci s'accompagne d'un étalement en périphérie qui rend l'espace périurbain hétérogène, donc disputé entre plusieurs usages. Ainsi, à travers le monde, 200 millions d'urbains pratiquent l'agriculture et approvisionneraient 800 millions d'urbains en denrées alimentaires. Cependant, l'étude de l'agriculture périurbaine est confrontée à un déficit de données statistiques. Des études de cas menées dans les grandes métropoles africaines révèlent que cette agriculture satisfait entre 20 et $50 \%$ de la consommation en produits maraîchers des urbains. En Ouganda par exemple, les habitants de Kampala vivant dans un rayon de 5 $\mathrm{km}$ du centre-ville produisent près de $20 \%$ des aliments de base consommés sur la zone (Lançon et al., 2014). 
37 Dans d'autres métropoles africaines, l'agriculture périurbaine est confrontée aux mêmes enjeux de maintien. A Bamako par exemple, le régime foncier des parcelles agricoles en milieu urbain se rapproche de ce qu'on peut observer au Sénégal. En effet, ces terres sont sous la contrainte d'une dualité entre droit coutumier et droit positif. Le premier domaine d'implantation du maraîchage relève du droit coutumier. Or, selon le droit positif malien, ce régime coutumier ne confère qu'un droit d'usage : la propriété est régie par le droit positif. Le maraîcher peut faire abandon de tous ses droits fonciers coutumiers en faveur des collectivités, établissements publics et des demandeurs de concession. Ceci explique que $75 \%$ des exploitants à Bamako ne sont pas propriétaires des espaces qu'ils cultivent (Zalle et al., 2003). Ainsi, à l'instar de Pikine, la fragilité du système agricole périurbain suscite la question de sa durabilité dans un contexte où la demande urbaine en produits frais est en pleine croissance.

\section{Conclusion}

L'étude des enjeux de l'agriculture périurbaine dans le bassin horticole de Pikine a procédé par l'interrogation des exploitants et des vendeurs des produits maraîchers. Les enquêtes révèlent un niveau de revenu très moyen chez les deux catégories d'acteurs. Cette précarité économique est doublée d'une précarité foncière liée au statut foncier des espaces maraîchers. Ces derniers sont la cible des projets d'infrastructures non-agricoles à cause de leur position stratégique entre le centre-ville dakarois et sa périphérie. Il apparaît dès lors que le maraîchage risque de disparaître si les dynamiques en cours continuent, ce qui risque de causer un préjudice économique et social chez les acteurs du maraîchage. En effet, nous avons montré l'importance des enjeux chez les producteurs et les vendeurs : ils concernent essentiellement le chômage et ses conséquences sur la stabilité des ménages touchés. Au regard de tous ces paramètres, nous pouvons affirmer que la disparition du maraîchage dans le bassin de Pikine serait un frein à l'emploi de plusieurs centaines de personnes, dont une majorité de pères et mères de famille. Le chômage engendré constituerait une charge supplémentaire pour des familles dont le revenu est déjà maigre, voire précaire.

\section{BIBLIOGRAPHIE}

BÂ A., MOUSTIER P. (2010), « La perception de l'agriculture de proximité par les résidents de Dakar », Revue d'Économie Régionale et Urbaine, 5, pp. 913-937.

BÂ A. (2007), Les fonctions reconnues à l'agriculture intra et périurbaine (AIPU) dans le contexte dakarois ; caractérisation, analyse et diagnostic de durabilité de cette agriculture et de son intégration dans le projet urbain de Dakar (Sénégal), Thèse de doctorat ès Sciences Agronomiques et de l'Environnement, AgroParisTech-UCAD, 356 p. et annexes.

BÂ DIAO (2004), «Situation et contraintes des systèmes urbains et périurbains de production horticole et animale dans la région de Dakar », Cahiers Agricultures, 13, 1, pp. 39-49. 
BRYANT Ch. R. (1997), «L'agriculture périurbaine : l'économie politique d'un espace innovateur », Cahiers Agricultures, 6, pp. 125-130.

CISSE I., FALL A.S. \& FALL S.T. (2001), « Caractéristiques de la zone des Niayes » in FALL et al., (dir.), Cités horticoles en sursis? L'agriculture urbaine dans les Niayes au Sénégal, Ottawa (Ontario), CRDI, pp 1-10.

DIOP Kh. (2015), Étude des contraintes du maraîchage dans la Grande Niayes de Pikine et leurs conséquences, Mémoire de Master 2 ès Lettres et Sciences Humaines, Université Gaston Berger de Saint-Louis, $75 \mathrm{p}$.

DIOP S.M., (2012), Analyse des impacts des activités anthropiques sur les ressources naturelles de la Grande Niaye de Pikine, Mémoire de Master 1 ès Lettres et Sciences Humaines, Université Gaston Berger de Saint-Louis, Sénégal, 82 p.

FALL S.T., FALL A.S. (2001), Cités horticoles en sursis ? L'agriculture urbaine dans les Grandes Niayes au Sénégal, Ottawa (Ontario), CRDI, 126 p. et annexes.

GAYE M., NIANG S. (2010), Manuel des bonnes pratiques de l'utilisation saine des eaux usées dans l'agriculture urbaine, Dakar, ENDA RUP, $131 \mathrm{p}$.

LANCON F. et al. (2014), « L'extension urbaine à travers le monde : enjeux pour les villes et les campagnes », Cahiers Déméter, 15, pp. 83-101.

MINISTERE DE L'AGRICULTURE ET DE L'ELEVAGE (2000), Recensement national de l'agriculture 19898-99. Volume 5, Répertoire des zones et sites de production horticole périurbains d'après le prérecensement horticole 1999-2000, République du Sénégal, 126 p.

MINISTERE DE LA SANTE, DE L'HYGIENE ET DE LA PREVENTION (2002), Décret $n^{\circ}$ 2002-1042 du 15 octobre 2002 ordonnant l'élaboration et la mise en cuvre du programme d'Actions pour la Sauvegarde et le Développement urbain des « Niayes » et zones vertes de Dakar et prescrivant des mesures de Sauvegarde, République du Sénégal, 3 p.

MOUSTIER P., PAGES J. (1997), « Le péri-urbain en Afrique : une agriculture en marge ? ", Economie rurale, 241, pp. 48-55.

NDAO M. (2012), Dynamiques et gestion environnementales de 1970 à 2010 des zones humides au Sénégal : étude de l'occupation du sol par télédétection des Niayes avec Djiddah Thiaroye Kao (à Dakar), Mboro (à Thiès) et Saint-Louis, Thèse de doctorat ès Géographie et Aménagement, Université de Toulouse 2 Le Mirail, cotutelle internationale avec l'Université Gaston Berger de Saint-Louis, Sénégal, 365 p. NDIONE E.S., GUEYE O. (2009), Pikine aujourd'hui et demain. Diagnostic participatif de la ville de Pikine (Dakar, Sénégal), Enda Graf Sahel et Afrique de l'Ouest, 30 p.

SMITH O.B., MOUSTIER P., MOUGEOT L.J.A. \& FALL A. (eds.) (2004), Développement durable de l'agriculture urbaine en Afrique francophone : Enjeux, concepts et méthodes, CIRAD-CRDI, 173 p.

ZALLE D. et al. (2003), Le foncier dans l'agriculture urbaine dans le district de Bamako, Centre Amadou Hampâté Bâ pour le Développement Humain durable et la qualité de vie, 27 p.

\section{NOTES}

1. Dakar, Thiès, Louga, Saint-Louis.

2. Avec l'élection de Me Abdoulaye WADE en mars 2000 qui a lancé « les grands projets du Chef de l'État ». 
3. Cette population est basée sur le recensement national de l'agriculture de 1998-1999. Le volume 5 est spécialement réservé aux "zones et sites de production horticole périurbains». L'utilisation de cette base de sondage est justifiée par l'absence de données ultérieures concernant la population de marâichers périurbains, ce qui justifie que les auteurs qui ont travaillé sur la question ont tous utilisé cette base : Bâ Diao (2004) ; Bâ, (2007); Bâ, Moustier (2010).

4. 1 FCFA $=0,0015200 €$

5. Programme de Renforcement et d'Équipement des Collectivités Locales, une initiative locale pour améliorer la desserte urbaine.

6. Dans le droit foncier coutumier des Wolofs, lamane veut dire «maitre de la terre ». Il est le responsable des terres familiales considérées comme indivises et inaliénables.

\section{RÉSUMÉS}

La croissance urbaine à l'échelle planétaire s'accompagne de défis tels que l'autonomisation, l'aménagement et la résorption du chômage en milieu urbain. En Afrique de l'Ouest et plus particulièrement dans la région des Niayes au Sénégal, le maraîchage emploie bon nombre de jeunes et de femmes. A Dakar, ces espaces agricoles subissent un recul depuis plusieurs années. Dans la Grande Niaye de Pikine qui constitue le plus grand bassin de production horticole de l'agglomération dakaroise, les maraîchers sont menacés par la pression foncière, malgré les enjeux liés au maintien de l'activité agricole.

Cette étude est une contribution à la connaissance des enjeux socio-économiques autour du maraîchage dans la Grande Niaye de Pikine. La méthodologie adoptée procède par des enquêtes auprès des maraîchers et des vendeurs de produits de la Grande Niaye.

Les résultats montrent une charge familiale importante chez ces deux catégories d'acteurs, mais également une précarité foncière des exploitations qui facilite les expropriations. Ceci menace l'équilibre de la chaîne de valeurs qui semble être entretenue par la proximité du bassin de production.

Urban growth on a global scale is accompanied by challenges such as empowerment, urban planning and the eradication of unemployment. In West Africa and more particularly in the Niayes region of Senegal, market gardening employs a large number of young people and women. In Dakar, these agricultural areas have been declining for several years. In the Great Niaye of Pikine, which is the largest horticultural production area in Dakar agglomeration, market gardeners are threatened by land pressure, despite the issues related to the maintenance of agricultural activity.

This study is a contribution to the knowledge of socio-economic issues around market gardening in the Great Niaye of Pikine. It proceeds through surveys of market gardeners and sellers of Grande Niaye products.

The results show significant family burdens for these two categories of actors, but also land precariousness of the farms that facilitate the expropriations. This threatens the balance of the value chain that seems to be maintained by the proximity of the production basin. 
INDEX

Keywords : Niayes, urban agriculture, socio-economic issues, market gardening, Senegal, Dakar Mots-clés : Niayes, agriculture périurbaine, enjeux socio-économiques, maraîchage, Sénégal, Dakar

\section{AUTEURS}

\section{KHALIFA DIOP}

Laboratoire Leïdi « Dynamique des territoires et développement », Université Gaston Berger de Saint-Louis du Sénégal, diopkhalifa21@yahoo.fr

\section{CHEIKH AHMED TIDIANE FAYE}

Laboratoire Leïdi « Dynamique des territoires et développement », Université Gaston Berger de Saint-Louis du Sénégal, chatifa@yahoo.fr

\section{SEYDOU ALASSANE SOW}

Laboratoire Leïdi « Dynamique des territoires et développement », Université Gaston Berger de Saint-Louis du Sénégal, sowseydoualassane@yahoo.fr 